\title{
Validation of Vehicle Model Response with an Instrumented Experimental Vehicle
}

\author{
Mohamad Hafiz Harun ${ }^{1, *}$, Mohd Razali Md Yunos ${ }^{1}$, and Muhamad Azwar Azhari ${ }^{1}$ \\ ${ }^{1}$ Faculty of Engineering Technology, Universiti Teknikal Malaysia Melaka, Malaysia
}

\begin{abstract}
A steering aid system called active steering is evaluated by simulating different kinds of driving events. The main purpose of the steering system is to allow the driver control the vehicle independently. A full car vehicle model is simulated in Matlab/Simulink with 14 degree of freedom of equations which include the ride vehicle model and also the handling model. The steering angle is the input of the vehicle model that should be focused on. The angle of the steering system between the tires when turning the vehicle is taken in consideration. Simulations are made on different road conditions effect and also side wind disturbances. Different values are applied to the simulation to reduce the effect of the driving events. Therefore, these simulations results to provide a better improvement to the steering system. The aim for this work is to validate the vehicle response with an instrumented experiemental vehicle. Specific driving events in these simulations are the road adhesions and lateral side wind disturbances.
\end{abstract}

\section{Introduction}

In an age of advanced technology, automobile is a life's necessity. It also acts as a transportation to travel for a distance no matter a short or long journey. Therefore, automobile had become more and more convenience nowadays. In this competitive field, vehicle must be improved in comfort and safety. By doing so, development of vehicles should be done to ensure new technology is implanted into the driving system to enhance driver pleasure.

A vehicle consists of many systems to maintain each of the vehicle parts function well. The steering system acts as one of the important system in the vehicle. For the past few decades, the development of steering system has tremendously experienced many stages and the steer by wire system is the latest technology of steering system for passenger cars. Steer by wire system are still not fully trusted by public consumers. On top of that, with the permission issues by the state regulations, AFS has become a customer choice. Active front steering system is the latest technology for passenger cars which implements an electronically controlled superposition of an angle to the hand steering wheel angle that is

* Corresponding author: mohamadhafiz@utem.edu.my 
prescribed by the driver. However, the permanent mechanical connection between steering and road remains $[1,2]$.

There are a lot studies focusing about the active steering system are carried out. But most of the studies focus on the stability of vehicle [3-8]. In active steering system, the driver will experience the variable steering gear ratio function at first and perceive the improvement of steering portability. AFS enables continuous and situation dependent variations of the steering ratio according to the vehicle's motion state, therefore AFS improve the manoeuvrability of the vehicle at low speed and the stability at high speed. The performances of stability improvement with active suspension system depend upon the quality of variation of the steering ratio to a certain extent. A controller of variable steering ratio for Active Front Steering system is designed, and virtual road tests are made in CarMaker driver vehicle-road simulation environment. The results of simulation tests validate the controller performance and the advantage of variable steering ratio function, also show that the driving comfort is improved at low speed especially, due to the Active Front Steering system alters the steering ratio according to the driving situation $[9,10]$.

The proposed AFS control structure is implemented on a validated full vehicle model. The full vehicle model can be approximately described as a 14 DOF system subject to excitation from braking and throttling inputs. It consists of 7-DOF vehicle ride model and 7-DOF vehicle handling model coupled with Calspan tire model [11]. The time used in this software was less compared to commercial program. Therefore, a more accurate result can be obtained.

This paper is organized as follows: The first section contains the introduction and the review of some related works, followed by the methodology of development of active front steering with affect of crossing wind test in the second section. The third section presents the result and discussion of the validation performance. The last section contains some conclusion.

\section{Methodology}

Computer simulations are carried out to verify the development of the active steering system in this study. For a basic start up, a model of the main component was developed. Vehicle Model of Mercedes E-Class was chosen as the simulation vehicle model. The characteristics of the Mercedes E-Class vehicle model was studied and simulated by Matlab/Simulink software. The stability test of the Mercedes E-Class was tested using crosswind test. Crosswind test is a wind test which is perpendicular or lateral to the vehicle itself. This affects the stability of the vehicle model when simulated in Carsim software.

In the analysis, crosswind test was applied on the vehicle model and changes of the vehicle are observed. The stability of the vehicle is focused and discussed. The driving stability is affected by the aerodynamic characteristics as well as the vehicle dynamic characteristics. This includes the yaw, lateral acceleration and longitudinal acceleration of the vehicle model. The vehicle dynamic characteristics is then plotted into graphs and compared. Different vehicle model will provide different graphs pattern. The other factors that affect the vehicle dynamic characteristics are the velocity of the wind.

The three vehicle dynamics characteristics act as an important role in the simulation. Longitudinal acceleration refers to the acceleration produces by the vehicle in a straight line. The lateral acceleration created when a vehicle tends to push a vehicle sideway and yaw rotation is the movement around the y axis. Stability test focused on these dynamic characteristics to improve the stability of the vehicle.

The validation of the ride and handling model is done by Matlab/Simulink software. The responses of the 14 DOF Mercedes E-Class vehicle models is compared with the 
instrumented experimental vehicle data using the same input conditions and data value. The dynamic response characteristics to be measured include lateral acceleration, longitudinal acceleration and yaw. Validation is done to obtain the graph result by two software which are the Carsim and Matlab software.

\section{Results and discussion}

Figure 1 below shows the lateral acceleration versus time. The dotted line represents the vehicle model simulation while the dark line depicts the Carsim model simulation. Both lines display similar pattern with different values. The duration of the simulation is set at 8 second. Each data was recorded at interval of 0.025 second. From Figure 1, data after 8 second is unrecorded as the lateral acceleration remains constant.

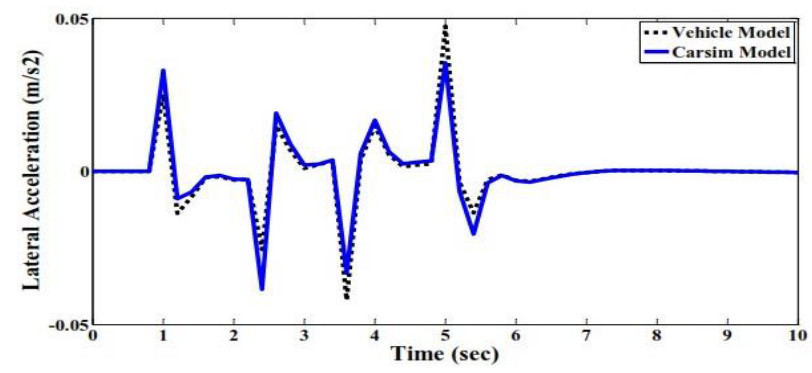

Fig. 1. Validation of lateral acceleration

The lateral acceleration is created when the vehicle is cornering. The acceleration tends to push the vehicle outwards. This is due to the centrifugal force created on the vehicle. Therefore, little acceleration is applied before reaching the apex of the curve. This is done to pull the vehicle through the curve. Therefore, the best way to take a sharp corner is to slow down before reaching the curve. Then, accelerate at the apex to turn the vehicle.

In this simulation, a vehicle model Mercedes E-Class was driven on a straight track with lateral side wind applied on it. Simulations showed that the vehicle speed with nonstationary wind excitation plays an important role in the crosswind stability of vehicle model. Therefore, the values obtained are slightly different compared to the actual values. As in real life, the speed of the wind and the wind turbulence will influence the crosswind stability. Actual measurement is hard to obtain from the vehicle model as simulation of vehicle model in Matlab/Simulink represents the vehicle model driven in real life. As for Carsim simulation, the speed of the wind is set ideally at both sides. It means that equal forces are applied side to side of the Mercedes E-Class vehicle model. As a result, perfect line graph can be obtained as true value of measurement is inserted to the Carsim software.

Figure 2 below illustrates the validation of longitudinal acceleration versus time. The range of the longitudinal acceleration is between $0.03 \mathrm{~m} / \mathrm{s}^{2}$ to $-0.04 \mathrm{~m} / \mathrm{s}^{2}$ and the maximum simulation time is 8 seconds. 


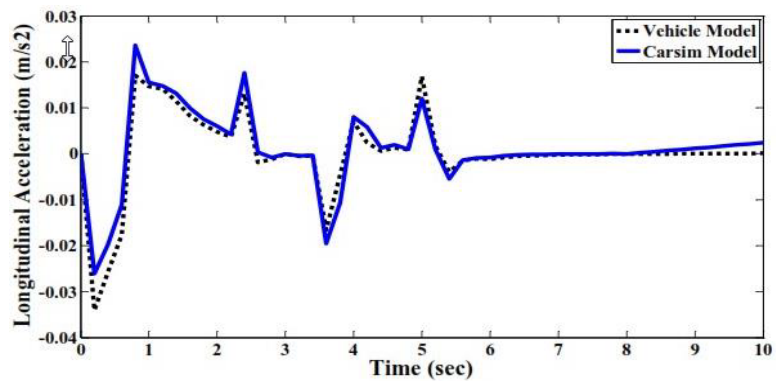

Fig. 2. Longitudinal acceleration

The longitudinal acceleration is the speed of a vehicle accelerates in a straight path. The positive value indicates acceleration whereas the negative value is for braking. A force felt in acceleration or braking movement around the horizontal axis of the vehicle is named as pitch.

First, the inconsistent pattern of the graph indicates the stability of the vehicle model. Pressure applied laterally to the vehicle will affect the movement of the vehicle. This also depends on the pressure value. A higher pressure will cause the stability of the vehicle to decrease. There are some factors that caused the value of both graphs to be asymmetry. Speed of the vehicle is one of the important factors to be discussed. Result cannot be compared between simulations and in real-life. As mentioned above, Carsim data is correct and cannot be denied. Therefore, the asymmetry data of Matlab/Simulink simulation is caused by the inconsistent speed driven on the vehicle. For example, an $80 \mathrm{~km} / \mathrm{h}$ and $100 \mathrm{~km} / \mathrm{h}$ speed value will produce different data when simulated with same lateral side wind condition. The value of $100 \mathrm{~km} / \mathrm{h}$ will higher than the value of $80 \mathrm{~km} / \mathrm{h}$ and the point in the graph will show significantly. Hence, speed of the vehicle may affect the stability of the vehicle even the weight of the Mercedes E-Class is one of the heaviest vehicles among luxury cars.

Figure 3 below represents the validation of yaw versus time on a same vehicle, Mercedes E-Class. The pattern of the graph goes smoothly compared to the previous graphs. The range of yaw degree is between 0.1 degree and -0.1 degree. The value remains constant zero for 0.0825 seconds.

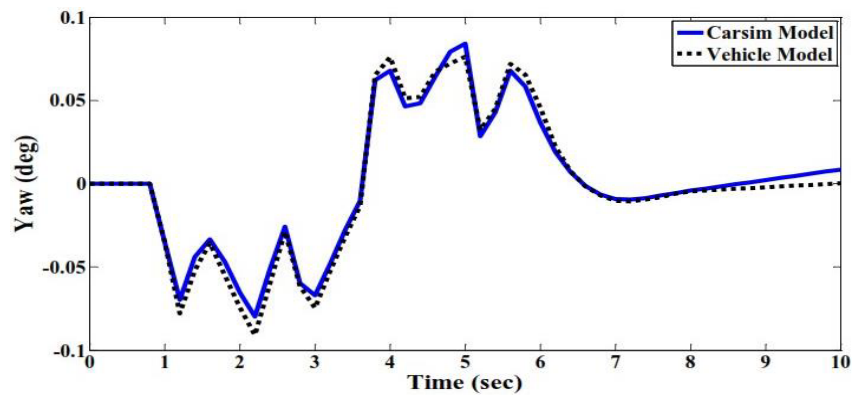

Fig. 3. Validation of Yaw

Yaw can be explained briefly as the ability to turn or rotate. A yaw rotation is the movement around the yaw axis of a rigid body. It changes the pointed direction either to the left or right of its direction of motion. Besides, yaw rate control is one of the main targets of electronic stability control systems that function to maintain the yaw rate of the vehicle. 
This is similar to the purpose of this analysis as the focus point is the stability of the vehicle.

The lateral side wind conditions show the actual stability of the vehicle. The vehicle model with $0.087007 \mathrm{~m} / \mathrm{s}^{2}$ positive yaw moment generates high yaw rate thus creates lower stability. The effect of surrounding also will affect the yaw rate, thus affect the stability of the vehicle. Higher wind force applied to the vehicle model will results the higher yaw rate. A rise and drop of the values runs by Matlab/Simulink software is due to the wind forces and side wind effect of the surroundings. Besides that, tire and suspension system of the vehicle act an important factors on the stability of the vehicle. Tire model can be categorized into many types. Each type may have different pressure and air density. Thus, different volume density of the tire may affect the stability result of the vehicle. Furthermore, flat tire do counted a part of it. The suspension system is the main stability system that stabilise the vehicle while having side conditions on roads. Nowadays, the Mercedes E-Class is using Airmatic air suspension system to improve the handling stability by reducing the vibrations and quieter actuation noises. With the advanced technology, stability may be improved.

\section{Conclusions}

The vehicle model is created by Matlab software. 14 DOF mathematical equations were developed in blocks. The 14 DOF includes the ride model and handling model. Both models acting the important part in this project to improve the vehicle stability due to the only factor lateral side wind effect. Therefore, validation graphs are compared in Matlab/Simulink simulations.

The author would like to acknowledge the Ministry of Science Technology and Innovation and Universiti Teknikal Malaysia Melaka for the support and funding throughout this study.

\section{References}

1. P. Koehn, M. Eckrich, Active steering-the BMW approach towards modern steering technology (SAE Technical Paper, 2004)

2. W. Klier, W. Reinelt, Active Front Steering (Part 1): Mathematical Modelling and Parameter Estimation (SAE Technical Paper, 2004)

3. C. Deling, Y. Chengliang, China Mechanical Engineering, 18, 3019 (2007)

4. X. Sun, J. Zhao, Tractor and Farm Transporter, 35, 91 (2008)

5. J. Guo, J. Li, Y. Li, Automobile Technology, 4 (2007)

6. Z. Yu, Z. Zhao, H. Chen, China Mechanical Engineering, 31, 652 (2005)

7. Q. Li, G. Shi, Y. Lin, W. Zhao, Automotive Engineering, 31, 629 (2009)

8. B. Lee, A. Khajepour, K. Behdinan, SAE 2006 World Congress, (2006)

9. J. Huang, J. Ahmed, A. Kojic, J.P. Hathout, Proceeding of the 2004 American Control Conference Boston, (2004)

10. Z. Gao, J. Wang, D. Wang, Procedia Eng., 15, 1030 (2011)

11. Z.A. Kadir, K. Hudha, M.Z.M. Nasir, M.R. Said, Proceedings of the International Conference on Plant Equipment and Reliability, (2008) 